\title{
Conditions for Generating Virtual Channels in Cochlear Prosthesis Systems
}

\author{
Charles T. M. Choi and Chien-Hua Hsu \\ Department of Computer Science and Institute of Biomedical Engineering, National Chiao Tung University, 1001 Ta Hsueh \\ Road, Hsin Chu 300, Taiwan, ROC
}

(Received 22 August 2008; accepted 10 December 2008; published online 16 December 2008)

\begin{abstract}
Simultaneous electrical stimulation of neighboring electrodes in cochlear prosthesis systems generates channel interaction. However, intermediate channels, or virtual channels between the neighboring electrodes can be created through controlled channel interaction. This effect may be exploited for sending new information to the hearing nerves by stimulating in a suitable manner. The actual stimulation sites are therefore not limited to the number of electrodes. Clinical experiments, however, show that virtual channels are not always perceived. In this paper, electrical simulation with finite element analysis on a half turn human cochlea model is adopted to model the virtual channel effect, and the conditions for generating virtual channels are discussed. Five input current ratios $(100 / 0,70 / 30,50 / 50,30 / 70,0 / 100)$ are applied to generate virtual channels. Three electrode arrays parameters are taken into consideration: distance between electrode contact and modiolus, spacing between adjacent electrode contacts and scale of electrode contact size. By observing the activating function contours, the virtual channel patterns and performances can be measured and examined. The results showed that a broad excitation pattern is necessary to produce the kind of electrode interaction that can form distinct virtual channels.
\end{abstract}

Keywords-Cochlear prosthesis, Cochlear implant, Virtual channel, Current steering, Electrical stimulation, Volume conduction model, Activating function, Finite element analysis.

\section{INTRODUCTION}

Cochlear implant (CI) technology has been developed for years to help people with profound hearing impairment to recover partial hearing. Typically, sound is captured by a microphone and is fed into a signal processor. The signal processor breaks the input signal into different frequency bands or channels and delivers the filtered signal to drive electrodes which are used to stimulate auditory nerve fibers. ${ }^{14,20}$ The number

Address correspondence to Charles T. M. Choi, Department of Computer Science and Institute of Biomedical Engineering, National Chiao Tung University, 1001 Ta Hsueh Road, Hsin Chu 300, Taiwan, ROC. Electronic mail: c.t.choi@ieee.org of spectral bands of stimulation is determined by the number of electrodes. Currently, there are only about 12-22 electrodes implanted into a CI user for a commercially available device while there are about 30,000 or more auditory nerve fibers for a typical human ear. The lack of spectral resolution due to the small number of electrode can contribute to poor speech perception in noise, music perception, and tonal language understanding. ${ }^{25}$

For speech processing strategies, electrical stimulation by two electrodes can produce two pitch components, but channel interactions are generated by stimulating individual electrodes simultaneously, ${ }^{3}$ which produce threshold changes consistent with instantaneous electric field summation. This will affect or distort the intended perception and usually is undesirable. However, experiments show that by exciting two adjacent electrodes simultaneously in a suitable manner, $2,9,10,15,17,22-24$ the CI users can perceive a pitch which is between two pitches perceived when the two electrodes are excited individually. The intermediate pitch is the so called virtual channel since there is no real electrode to generate a real stimulation channel there. The mechanism for controlling the virtual channels, called current steering, ${ }^{2,10,15,23}$ steers the current applied to adjacent electrodes in order to generate virtual channels according to the current ratio between the electrodes. This technique can be used to expand the spectral resolution without changing the implanted hardware or the number of CI electrodes.

The virtual channel can also be simulated by a modeling approach with the result being consistent with the experiment result. ${ }^{4,6}$ In our early work, ${ }^{5}$ virtual channel effect based on the finite element model with different electrode contact shape, was studied. In the study, planar, ball and half band electrode contacts were modeled, which covered the most popular design of electrode arrays then and now. The result showed that the examined shapes can create virtual channels normally; any difference in the effect due to the shape was not apparent. 
In clinical experiments, however, virtual channels are not always perceived, ${ }^{2,10,15,22-24}$ perhaps due to the individual hearing loss factor of each user or improper electrical stimulation from the electrodes. From the experiment results, it is found that virtual channels at the medial and apical electrode pairs are easier to perceive. ${ }^{10,15}$ Since the medial and apical electrodes are usually closer to the lateral wall of the cochlea, thus generating a larger current spread and more channel interaction. ${ }^{21}$ Clinical experiments show the possibility that the generation of virtual channels by electrical stimulation could be linked to the distance between the electrode contacts and the auditory nerves (modiolus).

Generally, the current design of the electrode array tends to decrease the channel interaction in order to provide the improved stimulation efficacy of individual electrodes, such as moving the electrode array closer to the modiolus (Contour array of Cochlear ${ }^{\mathrm{TM}}$ and Hifocus-helix array of Advanced Bionics Corporation) or enlarging the distance between adjacent electrode contacts (Medel $\mathrm{C} 40+$ array). However, since the virtual channel depends upon a suitable electrical interaction, the interaction free design mechanism might decrease the effect of the virtual channel and thus may affect the outcome of the clinical experiments. In this paper, a finite element model incorporating various electrode array configurations is used to study the conditions for generating virtual channels and to assess the virtual channel's effect in cochlear prosthesis systems. Although neural survival distribution has significant impact on the creation of virtual channels, it is beyond the scope of this paper and will be addressed in future studies.

\section{METHODS}

\section{Model of the Human Cochlea and Analysis Approach}

A half turn human cochlea model with a partial electrode array based on HiFocus-1j electrode array geometry from Advanced Bionics Corporation is used for study in this paper (Figs. 1 and 2). ${ }^{1}$ The cochlea model is constructed according to our early work, ${ }^{7}$ and is adapted to the dimension of scala tympani based on the study of Hatsuchika et al. ${ }^{12}$ The modeled cochlea is embedded in a bone, which is not shown in the figure, to form the complete model. Although the structure of a real cochlea is spiral and tapering, the basal turn, especially for the first half turn, is close to a circular shape with a similar dimension. The modeled cochlea

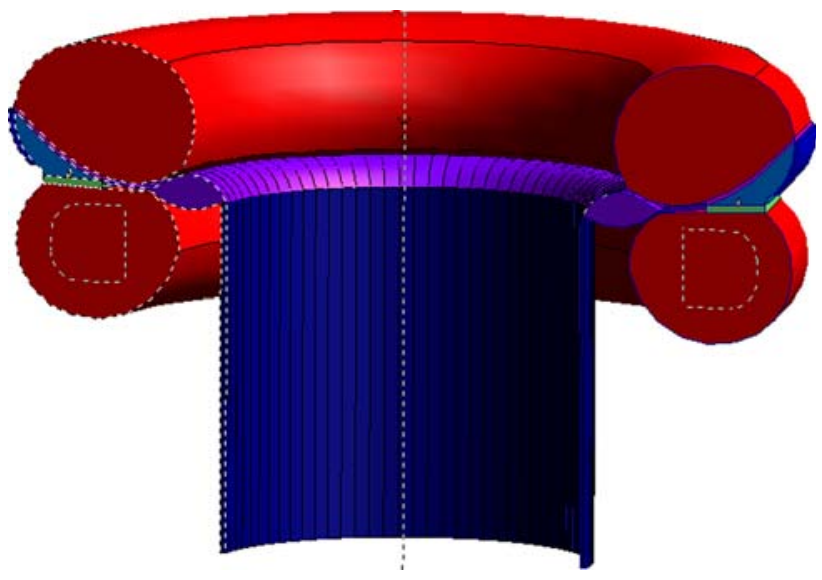

FIGURE 2. The finite element model of the cochlear implant system.
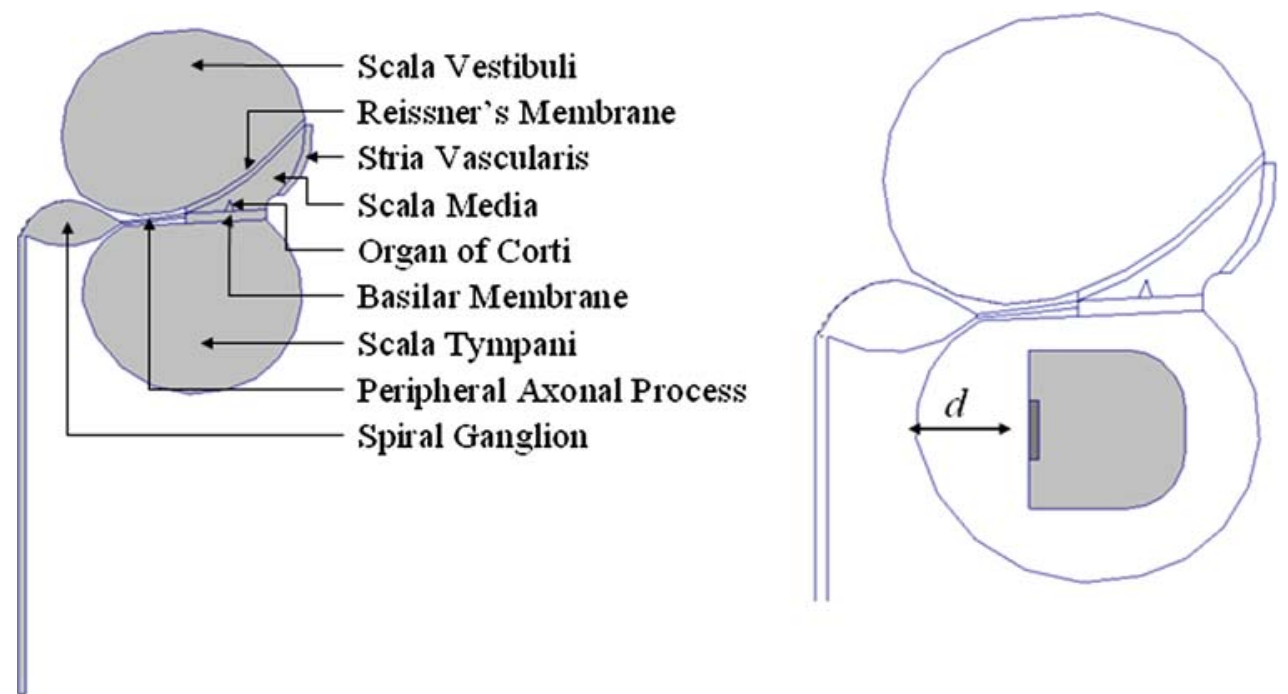

FIGURE 1. Cross-section of the cochlea model studied. The electrode array is located at a position of distance $d$ between the electrode contact surface and the inner wall of the scala tympani. 


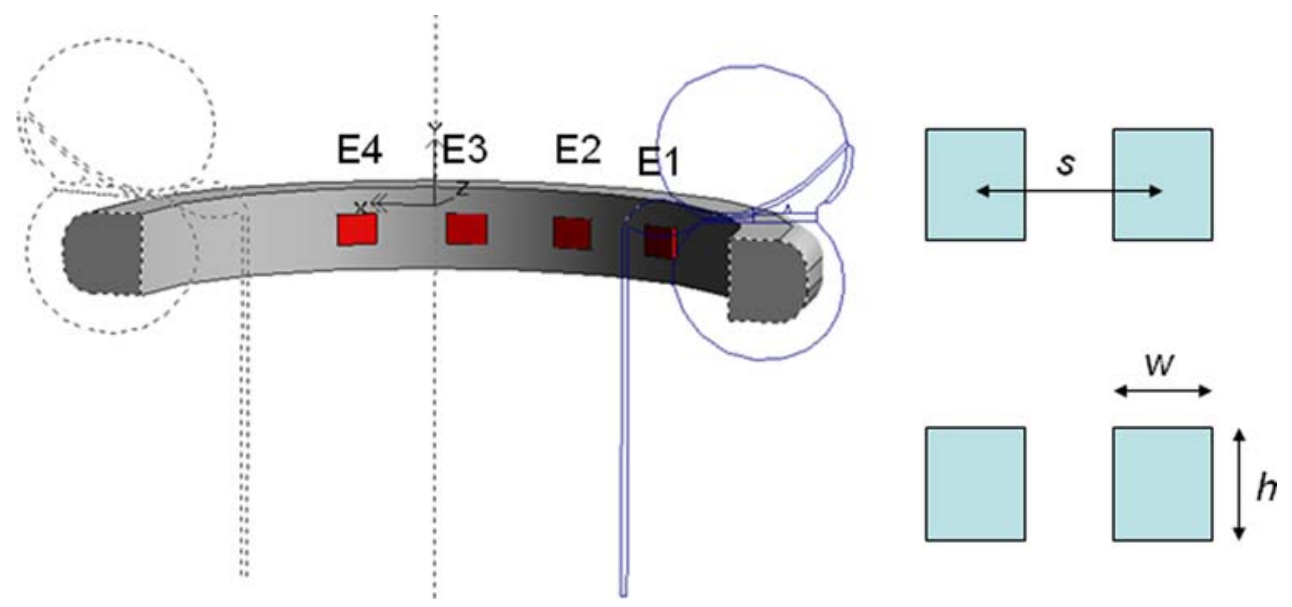

FIGURE 3. The electrode array of the cochlear implant model. The electrode contacts are spacing with various $s$ (centerto-center). The dimension of the electrode contact: height $h$ and width $w$.

is applicable to approximate the examination of neural excitation pattern for simulation. The modeled electrode array is placed inside the scala tympani (Fig. 3) as a typical CI system. The four electrodes are embedded into an insulated carrier, ex. silicone rubber, and only the contact surfaces are exposed to the tissue. The spacing between each electrode contact is $1.1 \mathrm{~mm}$ (center-to-center) and the dimension of each contact is $0.4 \mathrm{~mm}$ in width and $0.3 \mathrm{~mm}$ in height according to HiFocus- $1 \mathrm{j}$. The configuration of the electrode array is set to mono-polar and the central two electrode contacts (2nd and 3rd contacts of the electrode array) are used to generate virtual channels in this study. All the electrode contacts are organized to orient to the modiolus perpendicularly, as in general usage.

The finite element method is used to compute the potential distribution in the human cochlea model. ${ }^{7}$ The resulting mesh of the model shown in Fig. 4 contains 97,197 nodes and 554,192 tetrahedra. The material resistivity parameters used in this model are listed in Table 1. 8,11 The electrical potential from the nerve fiber model is extracted in order to calculate the activating function (AF) along the basilar membrane and nerve fiber. ${ }^{8,11,18,19}$ The AF is the forcing term in the Hodgkin-Huxley equation which calculates the variation of trans-membrane potential and determines the excitation for a nerve fiber. As a result, the initial trans-membrane potential response of the nerve fiber is proportional to the AF value. The result of the finite element model can be used to plot an AF contour which provides a global view for locating the virtual channel and evaluating its performance. ${ }^{8,11}$ Since $\mathrm{AF}$ is computed based on static electric potential, it cannot be used to investigate the effect of stimulation rate and stimulation pulse width. The effect of stimulation rate and stimulation pulse width have on virtual channel will be addressed in another study. Neural

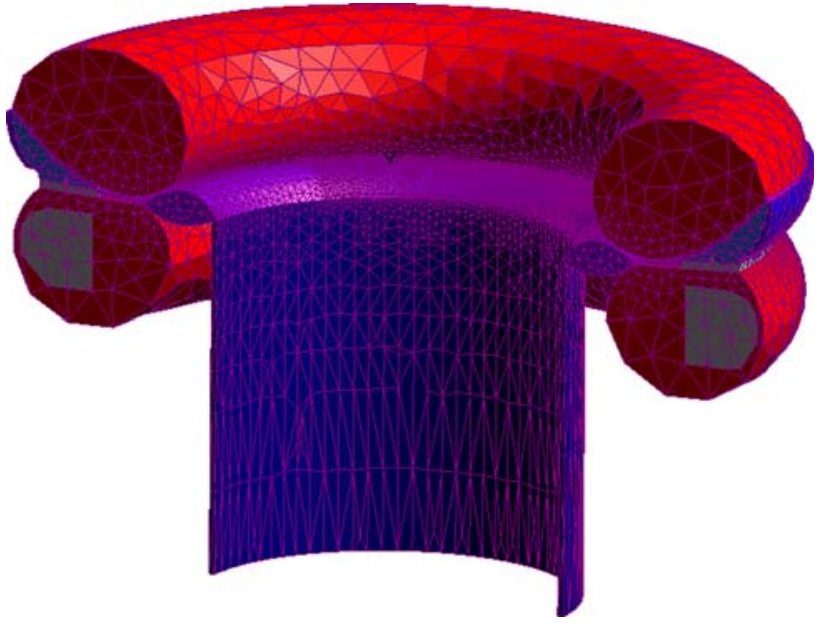

FIGURE 4. Mesh of the modeled cochlea which contains 97,197 nodes and 554,192 tetrahedra.

TABLE 1. Material properties for cochlea and electrode models.

\begin{tabular}{ll}
\hline Model component & \multicolumn{1}{c}{ Resistivity $(\Omega \cdot \mathrm{m})$} \\
\hline Electrode metal & 0.001 \\
Scala tympani and vestibuli & 0.7 \\
Scala media & 0.6 \\
Bone & 6.41 \\
Spiral ganglion & 3 \\
Peripheral axonal process (anisotropic) & 3 (axial) 15 (transverse) \\
Reissner's membrane & 340.13 \\
Basilar membrane & 4 \\
Stria vascularis & 125.79 \\
Organ of Corti & 83.333 \\
\hline
\end{tabular}

refractoriness ${ }^{16}$ and spike rate adaptation can produce temporal interaction in virtual channels, but this is beyond the scope of this paper and will also be addressed in another study. 


\section{Configurations of the Electrode Array}

Three electrode array parameters are examined for this study: distance between electrode contact and modiolus, spacing between adjacent electrode contacts and the scale of the electrode contact size. To examine the virtual channels, the electrical signals to be interacted are in phase. A ratio $\alpha$ of the input current is applied to the adjacent electrode pair and is normalized to five cases: $100 / 0,70 / 30,50 / 50,30 / 70,0 / 100$, which theoretically means that five distinct virtual channels can be generated.

\section{Distance Between Electrode Contact and Modiolus}

In the first simulation, the electrode array is placed at locations with various distances $d(0.9-0.4 \mathrm{~mm})$ from the inner wall of the scala tympani (Fig. 1). The range is adjusted according to the dimension and shape of both the electrode array and the scala tympani so that they fit each other well. Due to the large size of the electrode carrier which occupies the space near the outer region of the scala tympani, the positions of the electrode contacts are distributed in the medial and inner regions of the scala tympani. Generally, the exact position of the implant electrode array is not easily controlled and is different for each CI user after the electrode array insertion surgery. Typically, it is best to place the electrode array very close to the modiolus in order to provide a higher stimulation efficacy, a lower electrical interaction and an improved stimulation power. However, there is no study which examines the relationship with regard to the virtual channel effect and the distance of electrode contacts to the modiolus. This simulation aims to study this kind of configuration.

\section{Spacing Between Adjacent Electrode Contacts}

The second simulation is to examine the relationship with regard to the virtual channel effect and the spacing between adjacent electrode contacts. Due to the inertia and stress for the carrier of Hifocus- $1 \mathrm{j}$, the electrode array usually lies against the lateral wall. The electrode array is therefore placed at the outer region of the scala tympani $(d=0.8 \mathrm{~mm})$ for this study. Various spacing $s(0.8-1.3 \mathrm{~mm}$, center-to-center $)$ between adjacent electrode contacts which covers most of novel design of electrode array, ex. Hifocus-Helix, is organized to study the effect of this parameter (Fig. 3).

\section{Scale of Electrode Contact Size}

The study of this situation can be separated into two factors: height $h$ and width $w$ of the electrode contact (Fig. 3) with only one factor varied at a time. The electrode array is located at the outer region of the scala tympani $(d=0.8 \mathrm{~mm})$ as the consideration above shows. The spacing between adjacent electrode contacts is fixed at $1.1 \mathrm{~mm}$ (center-to-center). Due to the typical dimension of the scala tympani and the electrode array, the height $h$ varies in the range of $0.1-0.5 \mathrm{~mm}$ with the width ranging from 0.1 to $0.6 \mathrm{~mm}$.

\section{RESULTS}

In order to provide an efficient assessment of the virtual channel effect, several factors are examined. An AF contour provides an impression of the excitation pattern for the electrical stimulation and an AF profile extracted from an AF contour provides a comparison virtual channel pattern for the five cases of $\alpha$. Virtual channel positions, beam widths and the total current provide systematic comparisons; the details are illustrated in the following paragraph.

Figures 5 and 6 show the potential and the activating function (AF) contour from the nerve fibers for the electrode array located near the lateral wall at $d=0.9 \mathrm{~mm}$. The input current ratio $\alpha$ applied to the electrode pair is set to 50/50. According to the mechanism of electrical stimulation for nervous systems, ${ }^{18,19}$ the AF indicates the initial trans-membrane potential response of the nerve fiber; so the positive peak denotes the area of stimulation. Though the intermediate stimulation site can also be observed in the potential contour, AF is used to assess the virtual channel effect. The positive peak value of AF is normalized to $1 \times 10^{4} \mathrm{~V} / \mathrm{s}$ for all $\alpha$, which signifies an equal stimulation intensity to the nerve fiber. The squares below the $x$-axis denote the electrode contacts' position along the basilar membrane. An intermediate stimulation site between the electrode pair shown in the bottom of Fig. 6 shows that this is a virtual channel since no physical electrode contact is positioned there. In order to effectively compare the virtual channels with the $\alpha$, the $\mathrm{AF}$ at the nerve fiber node with a positive peak $\mathrm{AF}$ value along the basilar membrane is extracted, and the result forms the AF profile as shown in Fig. 7. From Fig. 7, five distinct virtual channels are generated which means that five different groups of nerve fibers are excited between the neighboring electrode contacts instead of the two generated in a typical stimulation strategy.

However, virtual channels are not always generated or easily distinguishable in locations of different distances $d$ from the simulation result. Figure 8 shows the AF contour for the electrode array located near the inner wall with $d=0.4 \mathrm{~mm}$. Two stimulation peaks appear corresponding to the positions of the two active electrodes along the basilar membrane. This result is 


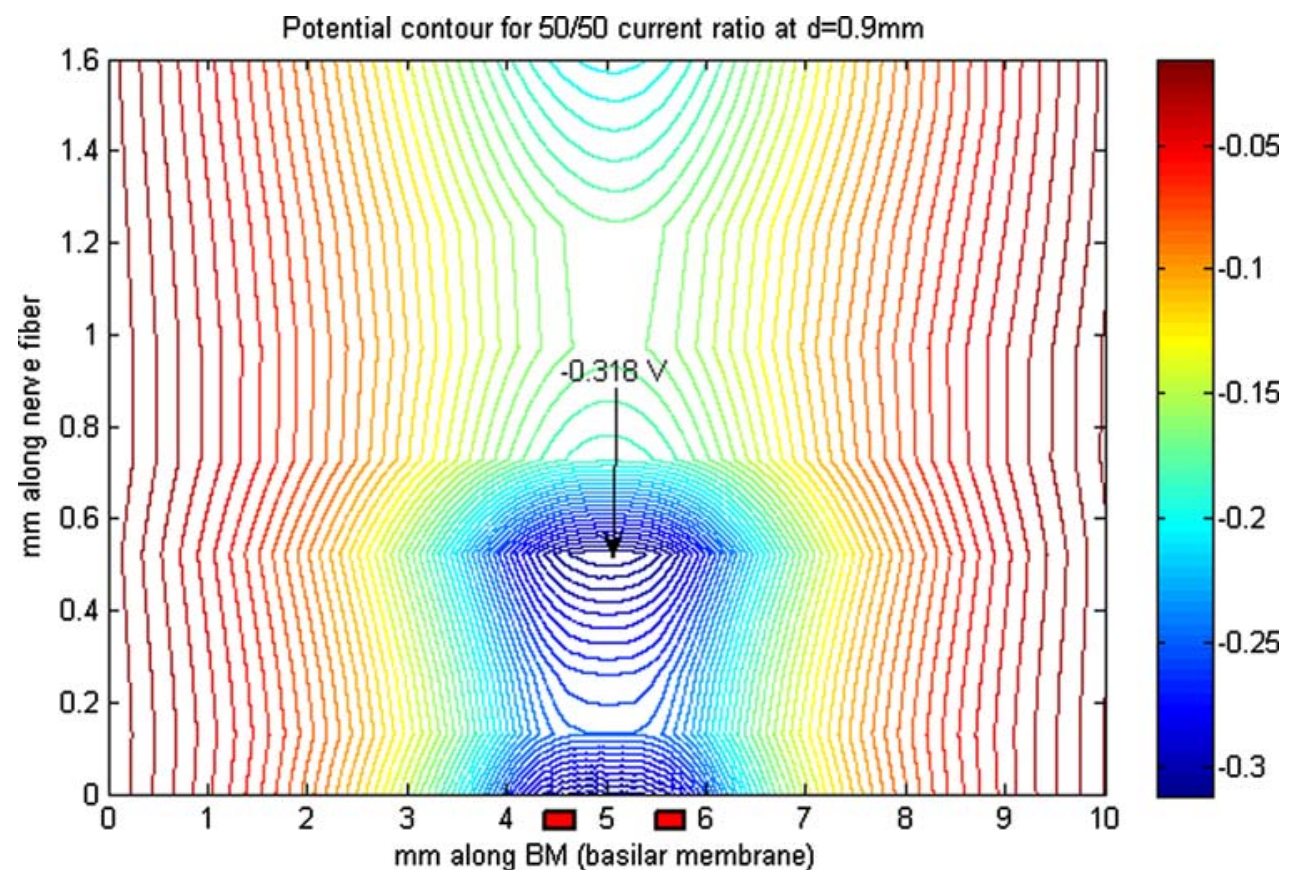

FIGURE 5. The potential contour for electrode array located against the lateral wall with $d=0.9 \mathrm{~mm}$. The squares below the $x$-axis denote the contacts position. The negative peak point $(-0.318 \mathrm{~V})$ is indicated by the arrow.

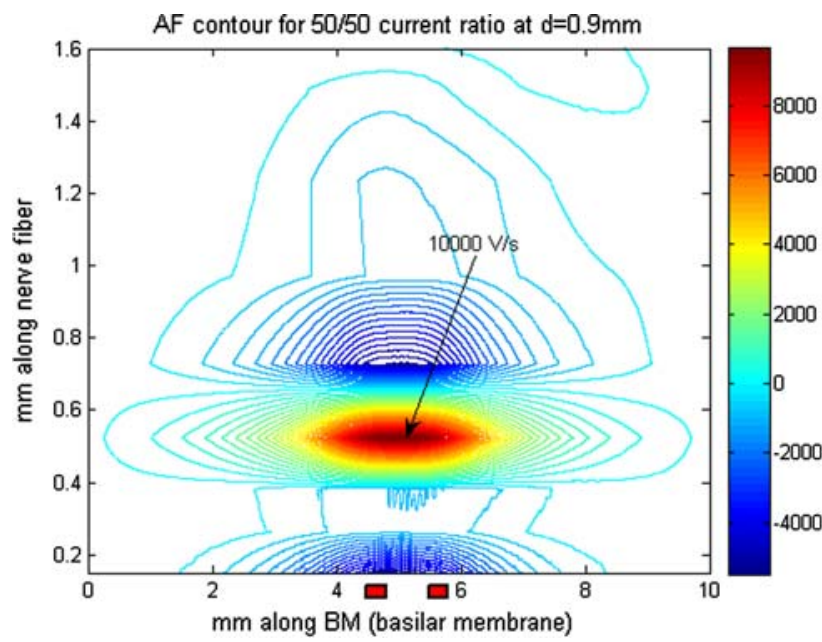

FIGURE 6. The AF contour for HiFocus-1j electrode array located against the lateral wall with $d=0.9 \mathrm{~mm}$ and current ratio $\alpha$ equal to $0.5(50 / 50)$. The squares below the $x$-axis denote the contacts position.

undesirable since it does not generate only one distinct virtual channel. The AF profile in Fig. 9 shows the five generated virtual channels tend to spread to the lateral sites which are the locations of the real electrode contacts. This makes the virtual channels indistinguishable.

By examining the distances $d$ (Fig. 10), it is found that when the electrode array is placed toward the lateral wall of the scala tympani $(d=0.7-0.9 \mathrm{~mm})$, the

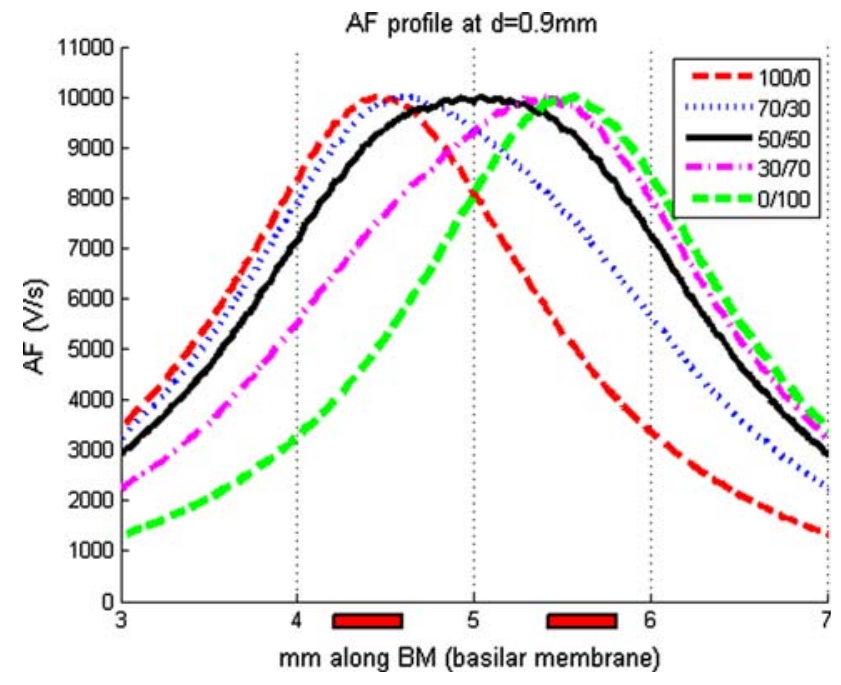

FIGURE 7. The AF profile of two electrodes stimulation configuration for the five cases of input current ratio $\alpha$ using HiFocus-1j electrode array.

virtual channels generated are more distinctive and the further the electrode array is from the modiolus, the more distinct the virtual channels are. When the electrode array is placed toward the inner wall of the scala tympani $(d=0.4-0.6 \mathrm{~mm})$, the generated virtual channels are indistinguishable; the closer the electrode array to the modiolus is, the less distinct the virtual channels are. This result is consistent with the modeling result from Briaire et al. ${ }^{4}$ 
Figure 11 provides the systematic comparison of the virtual channel effect for the different locations of the electrode array. Figure 11a shows the virtual channel position vs. the input current ratio $\alpha$. The virtual channel position is defined as the geometric center for the positive peak in the AF profile. It provides a view for distinguishing different virtual channels. It can be

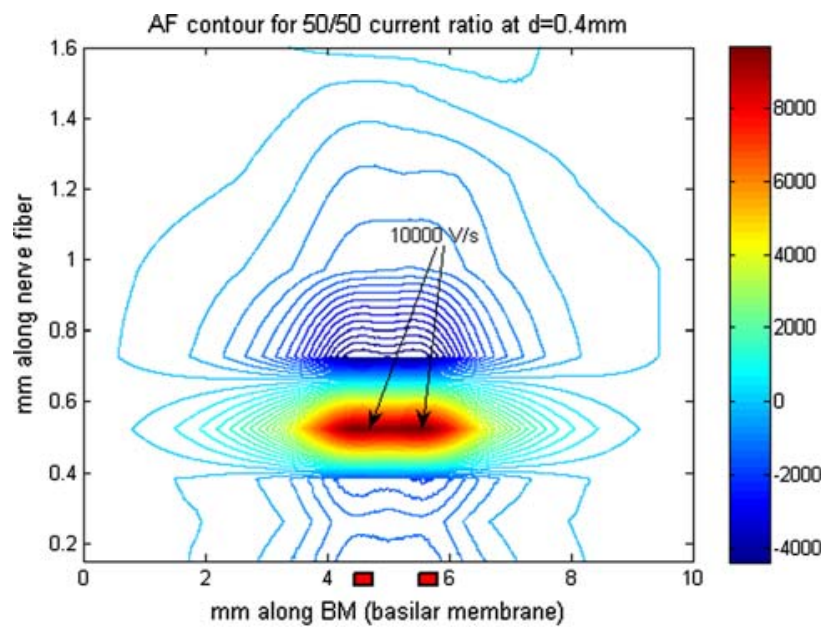

FIGURE 8. The AF contour for electrode array located near the inner wall with $d=0.4 \mathrm{~mm}$. There are two peaks indicating there are two sites of maximum stimulation. seen that when the electrode array is located near the lateral wall, the virtual channels varied more consistently with $\alpha$ (near linear distribution). However, when the electrode array is located near the inner wall, the virtual channels are hardly distinguishable and are distributed to the sites where the real electrode contacts reside. The linearity of the virtual channel position

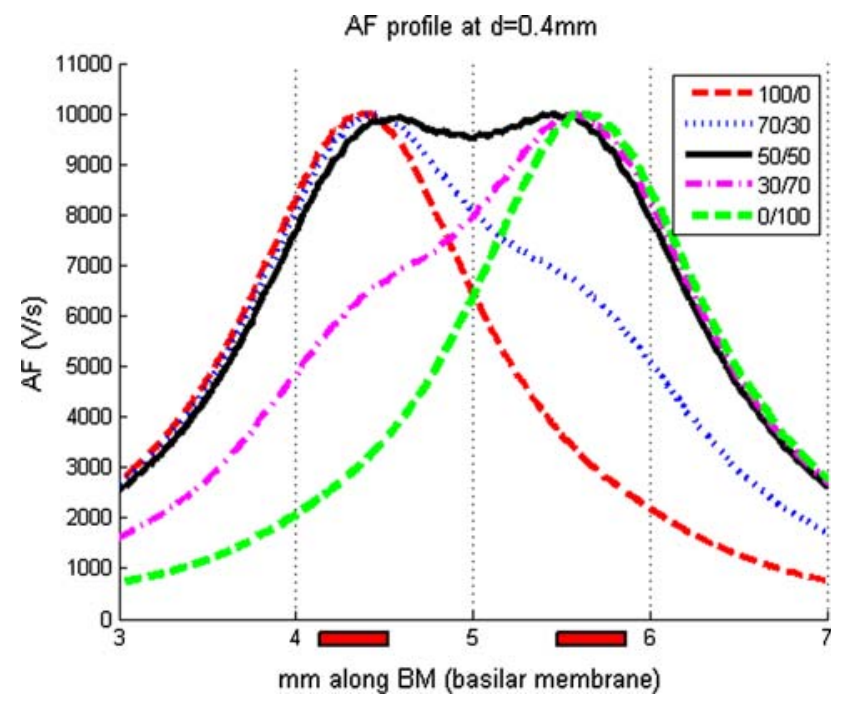

FIGURE 9. The AF profile for the five cases of input voltage ratio $\alpha$.

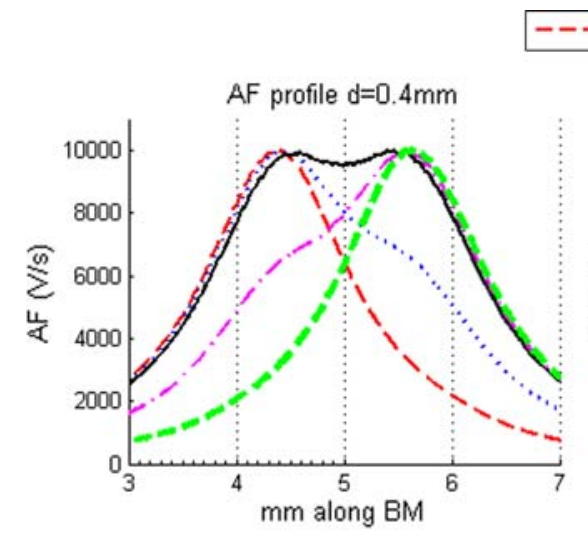

$$
---100 / 0 \cdots \cdots \cdot \cdots+70 / 30-50 / 50-\cdot-\cdots 30 / 70=-=0 / 100
$$
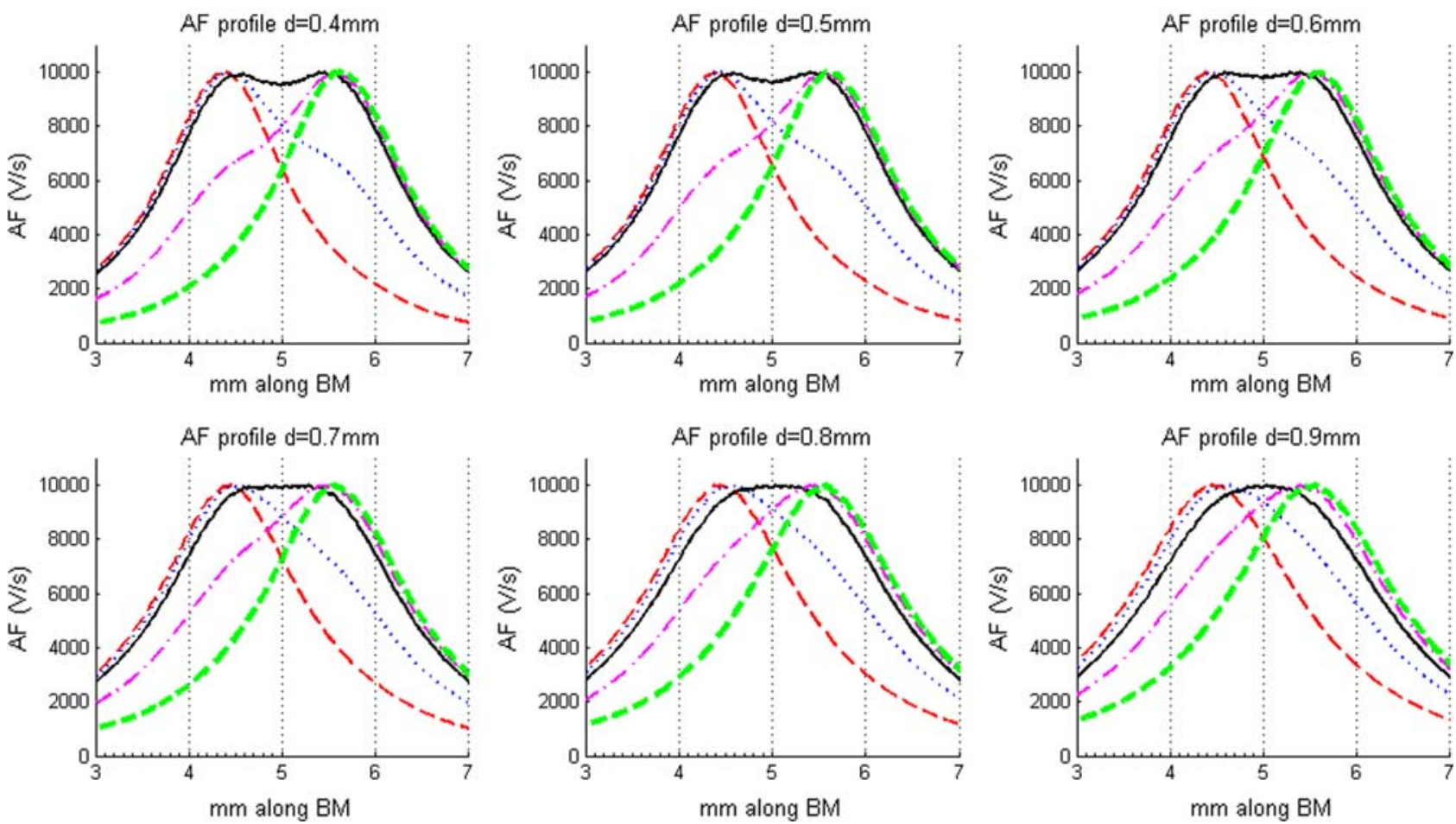

FIGURE 10. The AF profiles for all of the examined distances $d(0.4-0.9 \mathrm{~mm})$. 

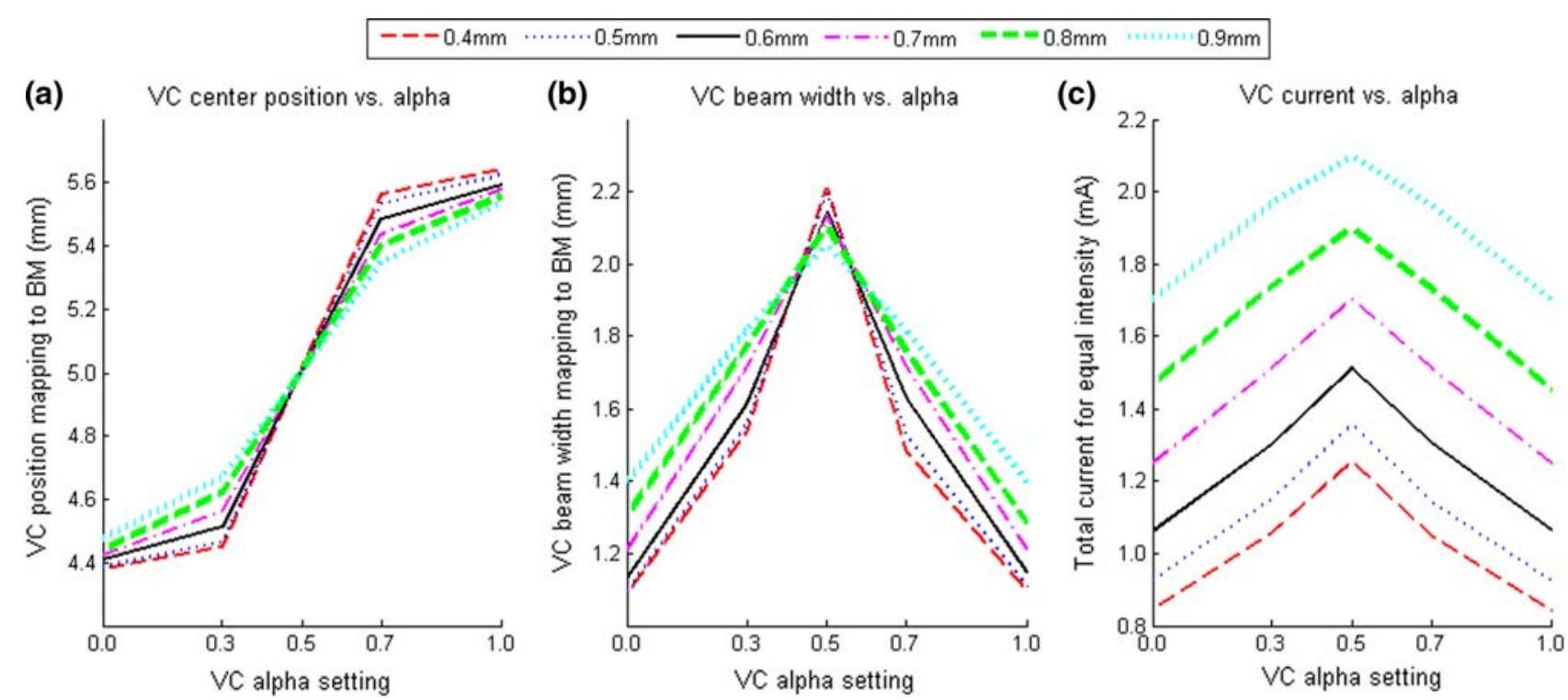

FIGURE 11. The comparison of the AF profiles for all of the examined distances $d(0.4-0.9 \mathrm{~mm})$. (a) VC center position vs. alpha; (b) VC beam width vs. alpha; (c) VC current vs. alpha.

distribution is important since it provides simplicity for controlling the virtual channel positions with the input current ratio.

Figure $11 \mathrm{~b}$ shows the virtual channel beam width vs. the input current ratio $\alpha$. For the sake of simplicity, the virtual channel beam width is defined as the width of the AF profile at the half power level along the basilar membrane. Though the actual beam width depends on the neural firing threshold, this can also enable a theoretical analysis. Typically, it is found that the narrower the beam width is, the more concentrated the virtual channels are. It is narrower for the virtual channels near the real electrode contacts since the stimulation there has better spatial selectivity. It can be observed that the virtual channel beam width is narrower for the electrode array located near the inner wall than when the array is near the lateral wall, since it is more concentrated when the electrode contact approaches the modiolus. It is the opposite for the intermediate channel due to the difficulty in generating virtual channels for the electrode array when located near the modiolus. Besides, the beam width is more uniform for all virtual channels when the electrode array is located near the lateral wall, in comparison to the higher variation of beam width when the array is located near the inner wall. Uniformity of beam width can provide a similar stimulation range for all virtual channels for CI users to perceive uniformly.

Figure $11 \mathrm{c}$ shows the total input current vs. the input current ratio $\alpha$. The total input current is input to bring the positive peak $\mathrm{AF}$ to the normalized value, i.e. $1 \times 10^{4} \mathrm{~V} / \mathrm{s}$, for all cases. The result shows that when the electrode array was placed near the lateral wall, the total current is higher since the current spreads out, which makes for inefficient stimulation to the nerve fibers. It can also be observed that the virtual channels near the center position need a higher current than do those near the real electrode contacts. Therefore, the intermediate channels need higher input current in order to generate equal electrical stimulation intensity. This is comparable to the modeling result from Litvak et al. ${ }^{13}$

The spacing between adjacent electrode contacts is another factor which influences the virtual channel effect. Figure 12 shows the AF profiles of all the examined spacing $s$ for the electrode array located at the outer region $(d=0.8 \mathrm{~mm})$. It can be observed that when $s$ is shorter, the virtual channels generated are more distinctive, but not so if it is wider. As the distance between electrode contact and the modiolus decreases, and the closer the adjacent electrode contacts (center-to-center), the more distinct the virtual channels are. In observing the channel position, beam width and total input current in Fig. 13, the comparison is more apparent. The channel position distribution is more linear and the beam width is more uniform and narrower when $s$ is shorter. The total current required is also smaller for shorter $s$ since more current interacts with closer electrode contacts to generate effective electrical stimulation.

For the simulation of the scale of electrode contact size, it is found that the relation between the virtual channel effect and scale of the electrode contact size is insignificant. This can be observed from Figs. 14a, 14b and $15 \mathrm{a}, 15 \mathrm{~b}$ for height and width examination. The channel position and beam width in the figures almost 


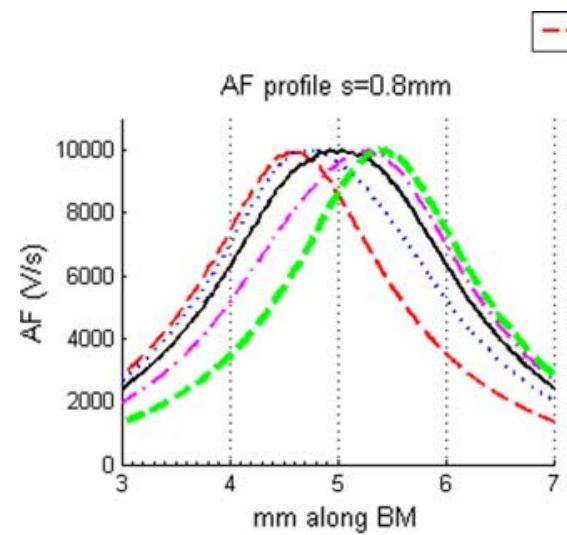

$$
---100 / 0 \cdots \cdots \cdots \cdot 70 / 30-50 / 50-\cdot-\cdot 30 / 70--=0 / 100
$$
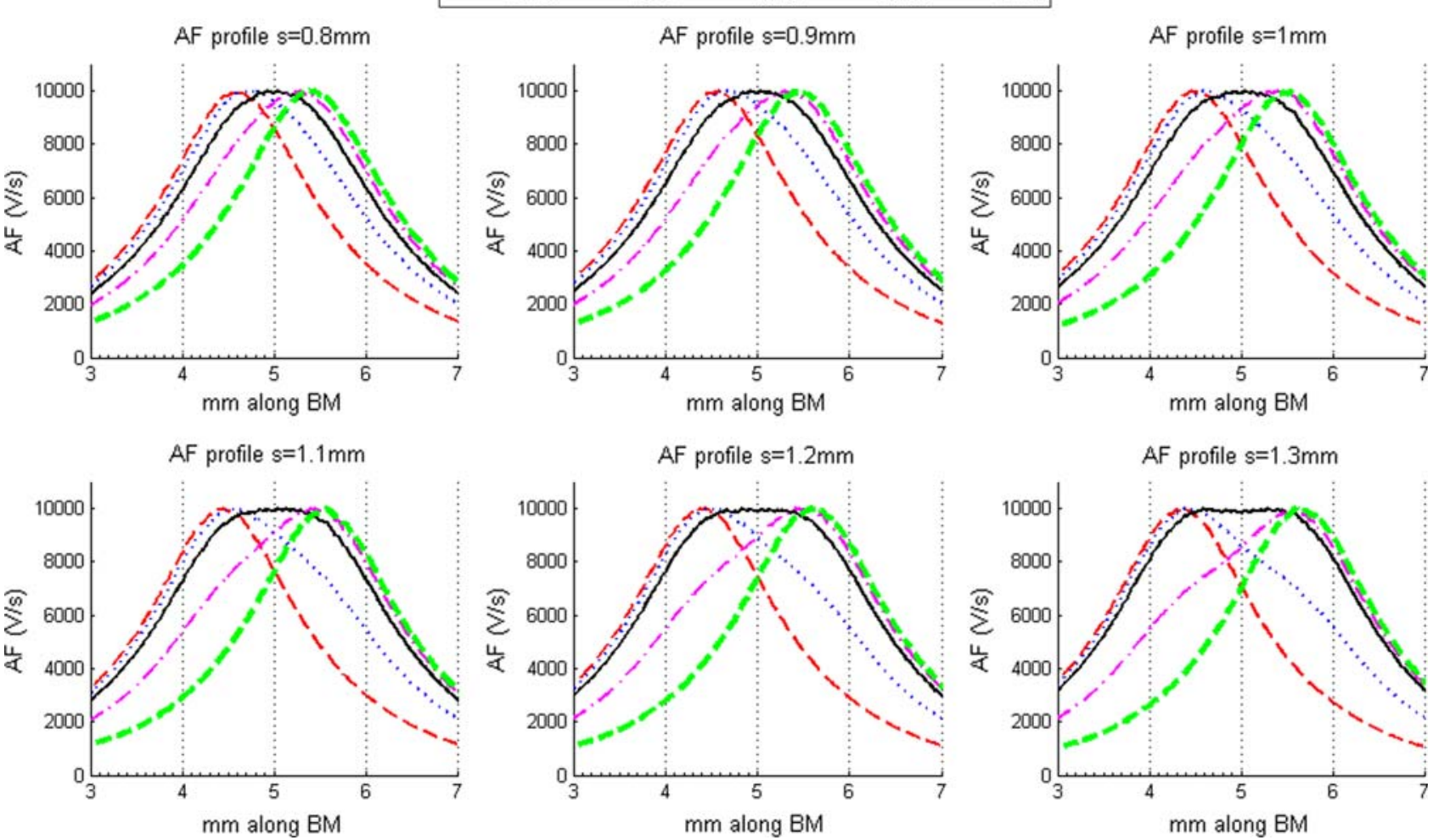

FIGURE 12. The AF profiles for all of the examined spacing $s(0.8-1.3 \mathrm{~mm})$. The electrode array is located at outer region of scala tympani $(d=0.8 \mathrm{~mm})$.

$-0.8 \mathrm{~mm} \cdots \cdots \cdots \cdot 0.9 \mathrm{~mm} \longrightarrow 1.0 \mathrm{~mm}-\cdot-\cdots 1.1 \mathrm{~mm}=--1.2 \mathrm{~mm} \cdots \cdots+1.3 \mathrm{~mm}$
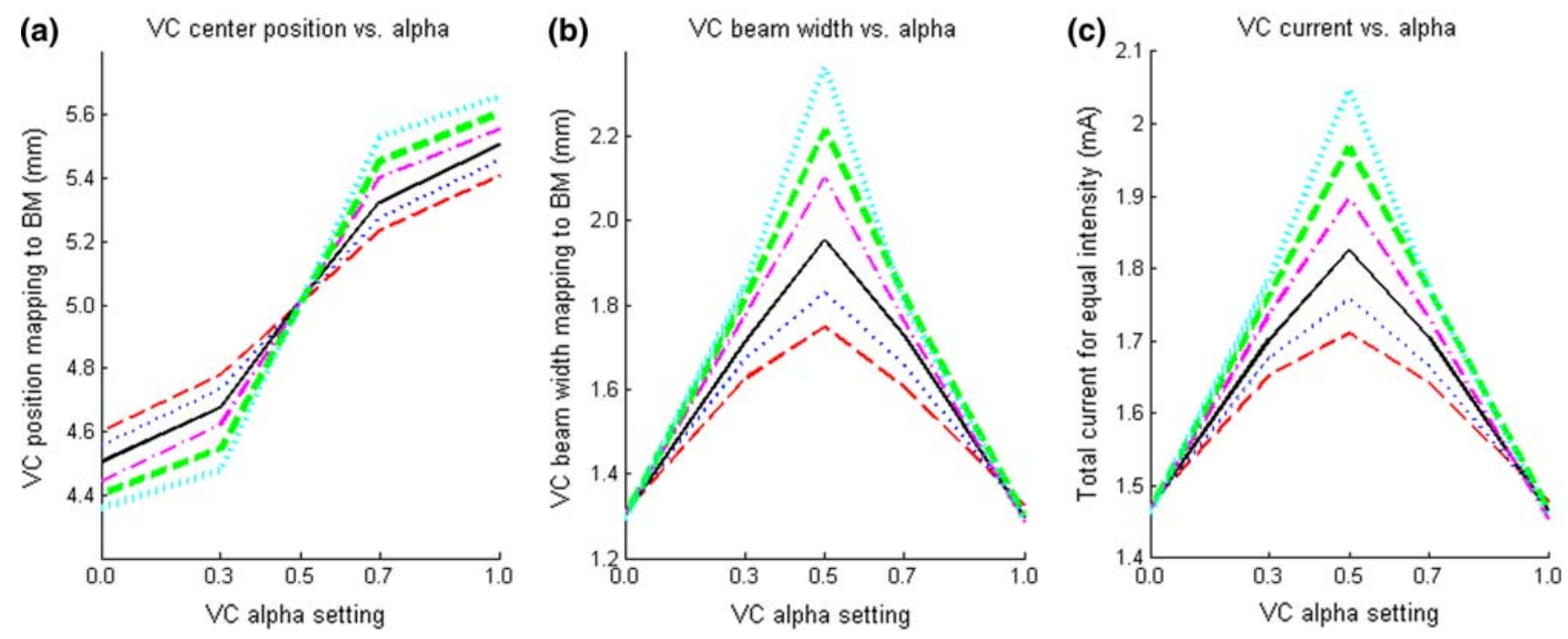

FIGURE 13. The comparison of the AF profiles for all of the examined spacing $s(0.8-1.3 \mathrm{~mm})$. (a) VC center position vs. alpha; (b) VC beam width vs. alpha; (c) VC current vs. alpha. The electrode array is located at outer region of scala tympani $(d=0.8 \mathrm{~mm})$.

overlap and are indistinguishable. The AF profiles are all very similar and therefore are not shown here. The only influence is the total input current. Figures $14 \mathrm{c}$ and $15 \mathrm{c}$ show the total input current applied to the smaller electrodes is lower than that applied to the larger ones, but the difference is not apparent. 


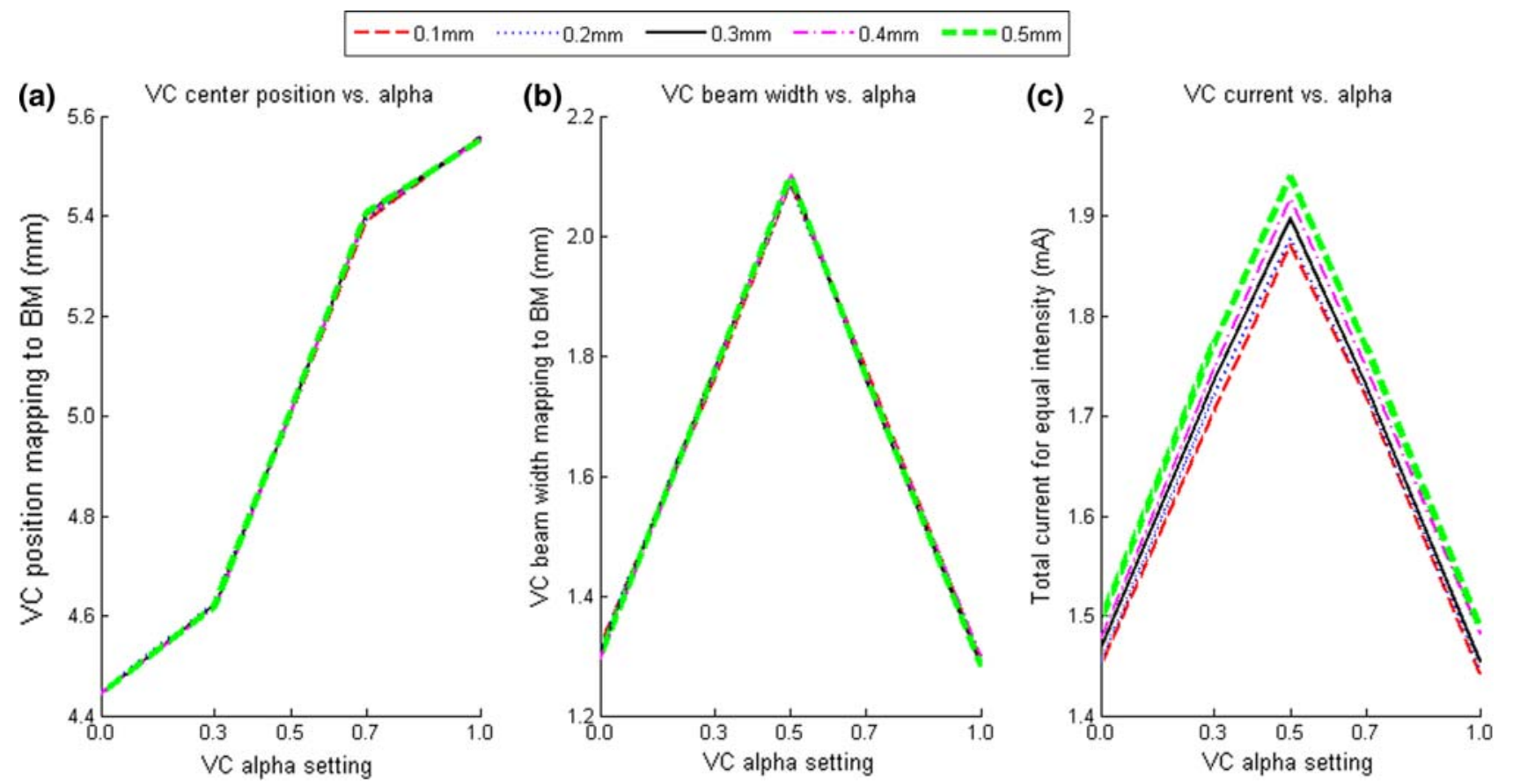

FIGURE 14. The comparison of the AF profiles for all of the examined height $h(0.1-0.5 \mathrm{~mm})$. (a) VC center position vs. alpha; (b) VC beam width vs. alpha; (c) VC current vs. alpha. The electrode array is located at outer region of scala tympani $(d=0.8 \mathrm{~mm})$.
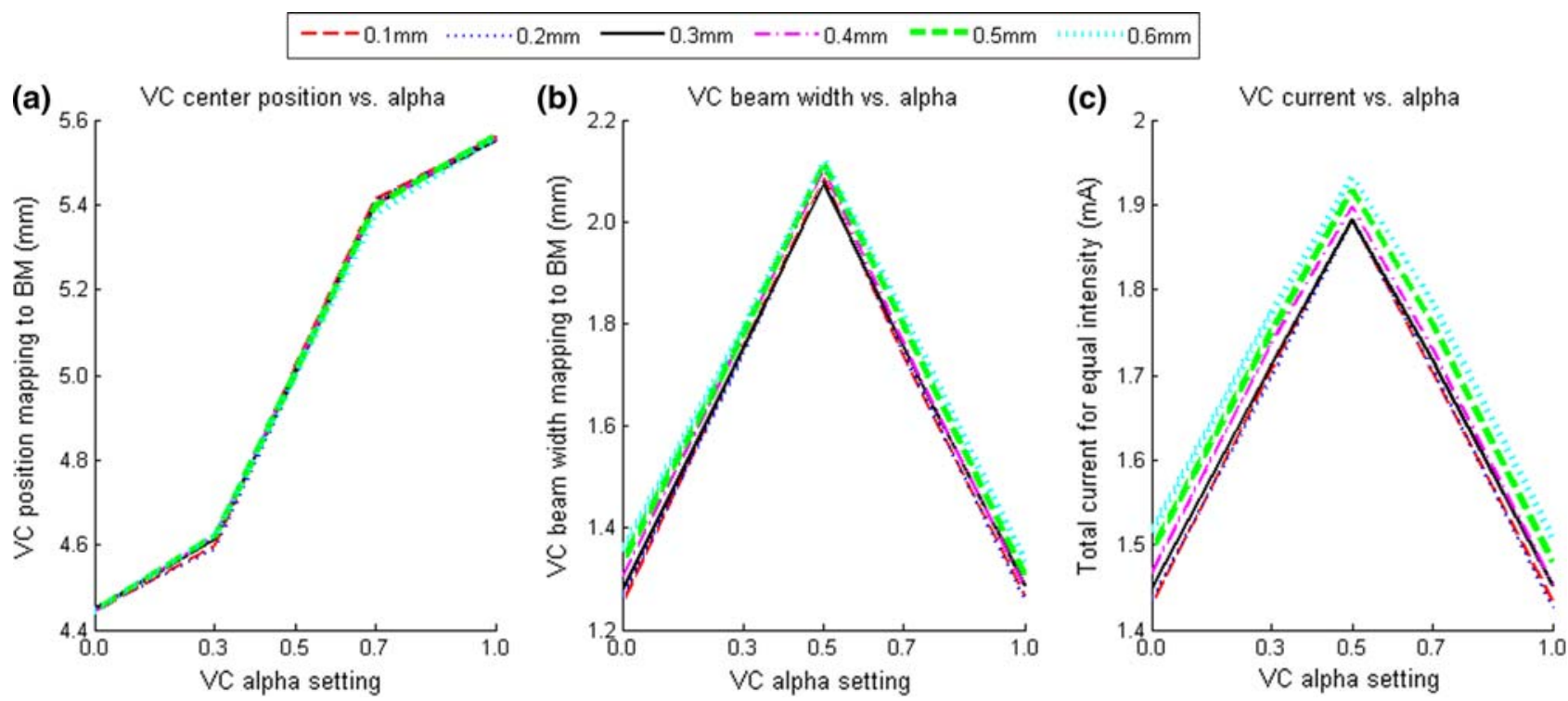

FIGURE 15. The comparison of the AF profiles for all of the examined width $w(0.1-0.6 \mathrm{~mm})$. (a) VC center position vs. alpha; (b) VC beam width vs. alpha; (c) VC current vs. alpha. The electrode array is located at outer region of scala tympani $(d=0.8 \mathrm{~mm})$.

\section{DISCUSSION}

This paper explored the conditions for generating virtual channels and assessed the virtual channel effect for various configurations of electrode arrays in cochlear prosthesis systems. The modeling approach and numerical analysis allowed us to study comprehensive conditions which are not achieved easily in clinical experiments or animal studies. The results provide a systematic view for virtual channels. The adoption of the dimension of an actual electrode array (ex. Hifocus-1j) made the study more accurate and realistic. 


\section{Clinical Experiment and Electrode Array Location}

Generally, it is better to have an electrode array with a large number of electrode contacts and place them very close to the modiolus. This can improve the spatial selectivity of stimulation and reduce channel interaction. It is possible to increase the effective number of electrode by adding virtual channels or virtual electrodes to the real electrodes, i.e. the physical number of electrode contacts. Since virtual channel depends on the electrical interaction of the stimulation and the efficacy of the stimulation, consequently, the degree of electrical interaction can affect the quality and the number of virtual channels generated. From the simulation results, the farther the electrode array is from the modiolus, the poorer the spatial selectivity and the better the results will be regarding the generation of more distinct virtual channels via electrical stimulation. This is consistent with the discussion on the cause of virtual channels, which showed that the virtual channels require suitable interaction between neighboring electrodes. More distinct virtual channels mean that the CI users can effectively perceive more number of channels, which is the goal of all auditory prostheses.

Due to the mismatch in size, curvature and shape of the electrode array inside the cochlea in different individual CI users, the spiral trajectory usually causes the electrode array to lie against the lateral wall of the cochlea, especially at the medial and apical parts. From the clinical experiment results, ${ }^{2,10,15,23}$ there are more distinct and perceptible virtual channels at the medial and apical electrode pairs than those at the basal electrode pair. Although those experiments did not provide data on where the electrode array is located, if the electrode array is indeed located closer to the lateral wall in the medial and apical parts, this is in consistent with our modeling result.

\section{Electrical Potential Distribution and Interaction}

Typically, the spacing between electrode contacts is designed wide enough to prevent electrical interaction. However, from the electrical point of view, the potential distribution is inversely proportional to the distance. The power is mainly distributed around the electrical source, i.e. the electrode contact, and decays rapidly as the distance increases. When two electrode contacts are spaced far apart, the potential contributed by the two electrode contact between the two electrode contacts will be less due to the larger distance. On the other hand, when two electrode contacts are spaced close enough, the potential from the two electrode contacts can interact to generate an intermediate channel which is the so-called virtual channel. From the simulation result, the effect of the virtual channels is not apparent when the spacing between electrode contacts is further apart, but it does improve the virtual channel performance when the spacing is closer.

\section{CONCLUSION}

A modeling approach with finite element analysis is proposed to study the conditions for generating virtual channels. The modeling result provides several directions for the electrode design to improve the virtual channels. First, the farther the electrode contacts are from the modiolus, it will be easier to produce the kind of electrode interaction that can form distinctive virtual channel. Second, the closer the adjacent electrode contacts are, the stronger the electrical interaction creating more of the virtual channel effect. The dimensions of the electrode contact have small impact on the virtual channels generation. All these simulation results can be used as reference and electrode design rules for future studies on virtual channels.

\section{ACKNOWLEDGMENTS}

This research was supported in part by the National Science Council of the Republic of China under contract number: NSC95-2221-E-009-366-MY3 and National Health Research Institute of Republic of China under grant: NHRI-EX97-9735EI.

\section{REFERENCES}

\footnotetext{
${ }^{1}$ Advanced Bionics Corporation. HiRes 90K: surgeon's manual for the HiFocus Helix and HiFocus $1 \mathrm{j}$ electrodes, 2005.

${ }^{2}$ Advanced Bionics Corporation. Increasing spectral channels through current steering in HiResolution bionics ear users, 2005.

${ }^{3}$ Balthasar, C., C. Boex, G. Cosendai, G. Valentini, A. Sigrist, and M. Pelizzone. Channel interactions with high-rate biphasic electrical stimulation in cochlear implant subjects. Hearing Res. 182:77-87, 2003. doi:10.1016/S03785955(03)00174-6.

${ }^{4}$ Briaire, J., R. Kalkman, and J. Frijns. Current steering: new model insights. Poster presented at the Proceedings of the 9th International Conference on Cochlear Implants, Vienna, Austria, 2006.

${ }^{5}$ Choi, C. T. M., and C. H. Hsu. Models of virtual channels based on various electrode shape. In: Proceedings of the 6th Asia Pacific Symposium on Cochlear Implants and Related Sciences, Sydney, 2007.

${ }^{6}$ Choi, C. T. M., and W. D. Lai. Modeling the virtual channels of the cochlear implant systems. In: Proceedings of the IEEE Conference on Electromagnetic Field Computation (CEFC 2006), Miami, Florida, USA, 2006.
} 
${ }^{7}$ Choi, C. T. M., W. D. Lai, and Y. B. Chen. Optimization of cochlear implant electrode array using genetic algorithms and computational neuroscience models. IEEE Trans. Magn. 40(2):639-642, 2004. doi:10.1109/TMAG. 2004.824912.

${ }^{8}$ Choi, C. T. M., W. D. Lai, and Y. B. Chen. Comparison of the electrical stimulation performance of four cochlear implant electrodes. IEEE Trans. Magn. 41(5):1920-1923, 2005. doi:10.1109/TMAG.2005.846228.

${ }^{9}$ Donaldson, G. S., H. A. Kreft, and L. Litvak. Place-pitch discrimination of single- versus dual-electrode stimuli by cochlear implant users. J. Acoust. Soc. Am. 118(2):623-626, 2005. doi:10.1121/1.1937362.

${ }^{10}$ Firszt, J. B., D. B. Koch, M. Downing, and L. Litvak. Current steering creates additional pitch percepts in adult cochlear implant recipients. Otol. Neurotol. 28:629-636, 2007. doi:10.1097/01.mao.0000281803.36574.bc.

${ }^{11}$ Hanekom, T. Three-dimensional spiraling finite element model of the electrically stimulated cochlea. Ear Hearing 22:300-315, 2001. doi:10.1097/00003446-200108000-00005.

${ }^{12}$ Hatsuchika, S., R. K. Shepherd, Y. C. Tong, G. M. Clark, and S. Funasaka. Dimensions of the scala tympani in the human and cat with reference to cochlear implants. Ann. Otol. Rhinol. Laryngol. 99(11):871-876, 1990.

${ }^{13}$ Litvak, L. M., A. J. Spahr, and G. Emadi. Loudness growth observed under partially tripolar stimulation: model and data from cochlear implant listeners. J. Acoust. Soc. Am. 122(2):967-981, 2007. doi:10.1121/1.2749414.

${ }^{14}$ Loizou, P. Mimicking the human ear. IEEE Signal Process. Mag. 15(5):101-130, 1998. doi:10.1109/79.708543.

${ }^{15}$ Luk, B. P. K., T. K. C. Wong, and M. C. F. Tong. Increasing spectral bands using current steering in cochlear implant users. In: Proceedings of the 6th Asia Pacific Symposium on Cochlear Implants and Related Sciences, Sydney, 2007.
${ }^{16}$ Mino, H., and J. T. Rubinstein. Effects of neural refractoriness on spatio-temporal variability in spike initiations with electrical stimulation. IEEE Trans. Neural Syst. Rehabil. Eng. 14(3):273-280, 2006.

${ }^{17}$ Oguz, P., and P. C. Loizou. Pitch perception using virtual channels. In: Proceedings of the Conference on Implantable Auditory Prostheses, Asilomar, Monterey, CA, 2001.

${ }^{18}$ Rattay, F. Analysis of models for extracellular fiber stimulation. IEEE Trans. Biomed. Eng. 36(7):676-682, 1989. doi:10.1109/10.32099.

${ }^{19}$ Rattay, F. The basic mechanism for the electrical stimulation of the nervous system. Neuroscience 89(2):335-346, 1999. doi:10.1016/S0306-4522(98)00330-3.

${ }^{20}$ Spelman, F. A. The past, present, and future of cochlear prostheses. IEEE Eng. Med. Biol. 18(3):27-33, 1999. doi: $10.1109 / 51.765186$

${ }^{21}$ Stickney, G., P. C. Loizou, L. N. Mishra, P. F. Assmann, R. V. Shannon, and J. M. Opie. Effects of electrode design and configuration on channel interactions. Hearing Res. 211:33-45, 2006. doi:10.1016/j.heares.2005.08.008.

${ }^{22}$ Townsend, B., N. Cotter, D. Van Compernolle, and R. L. White. Pitch perception by cochlear implant subjects. J. Acoust. Soc. Am. 82(1):106015, 1987.

${ }^{23}$ Trautwein, P. HiRes with Fidelity 120 Sound Processing: Implementing Active Current Steering for Increased Spectral Resolution in CII BionicEar ${ }^{\circledR}$ and HiRes90K Users. Valencia, CA: Advanced Bionics Corporation, 2006.

${ }^{24}$ Wilson, B. S., and M. F. Dorman. Cochlear implants: current design and future possibilities. J. Rehabil. Res. Dev. 45(5):695-730, 2008.

${ }^{25}$ Zeng, F. G., K. Nie, G. S. Stickney, Y. Y. Kong, M. Vongphoe, A. Bhargave, C. Wei, and K. Cao. Speech recognition with amplitude and frequency modulations. Proc. Natl. Acad. Sci. USA 102(7):2293-2298, 2005. doi:10.1073/pnas.0406460102. 